Levantamento florístico da região de São José do Rio Preto:

\title{
1. ${ }^{\text {a }}$ Contribuição ${ }^{1}$
}

\author{
PAULO NOGUEIRA DE CAMARGO? \\ GIÓRGIO DE MARINIS 3
}

1 - Realizado com o auxilio da Fundação de Amparo à Pesquisa do Est. de São Paulo, apresentado para publicação em 17-11-66; 2 - Cadeira de Botánica da ESALQ; 3 - Cadeira de Botánica da Fac. Filosofia, Ciências e Letras de S. J. do Rio Prêto. 


\section{RESUMO}

E apresentada uma primeira lista de plantas vasculares coletadas pelos A. A. na região de São José do Rio Preto (S. P.). O clima da cidade é mesotérmico, com tendência para megatérmico, correspondendo ao tipo Cwa de Kōppen. O solo predominante é um latossol vermelho-escuro, originado pela decomposição do Arenito de Bauru (Cretáceo superior). Entre as 76 espécies apresentadas nesta primeira lista, contam-se 23 próprias do cerrado (das quais, 14 exclusivas) e 20 invasoras (arvenses ou ruderais), distribuidas em 76 gêneros e 34 famílas.

\section{INTRODUÇÃO}

A Flora da Região de São José do Rlo Preto é pouco conhecida, sendo, até hoje, mal representada no Herbário do Instituto de Botânica de São Paulo (MATTOS), 1963)(*). Seu estudo constitui, portanto, uma contribuição valiosa para o conhecimento da Fitogeografia do Estado, e, ao mesmo tempo, fornece as bases indispensáveis para pesquisas ecológicas e fitossociológicas. Estas, por sua vez, poderão trazer interessintes subsídios para a interpretação do ambiente desta região, em seus aspectos climáticos, edáficos e bióticos.

$E$ É evidente a importância de tais estudos, do ponto de vista econômico-social, pois que, hoje em dia, a Ecologia já deixou de ser um simples capítulo da Biologia pura, para se tornar uma ciência de aplicação econômica. No Estado de São Paulo, por exemplo, as Faculdades de Filosofia, Ciências e Letras da Capital e de Rio Claro já estão, há anos, pesquisando os divensos aspectos ecológicos dos "cerrados", zonas estas até hoje de reduzida expressão econômico-șocial, $\epsilon$ cujo aproveitamento é de grande interêsse para o Estado, em virtude de sua notável extensão e privilegiada situação geográfica.

São José do Rio Preto, centro de uma ampla zona geográfica, ocupada em sua maior parte por "cerrados", constitui local especialmente indicado para pesquisas desta natureza, ainda mais que o mais próximo centro de estudo botânicos sitúa-se em Rio Claro, aproximadamente a 280 quilômetros de distância.

(*) Correspondência pessoal, de J. R. MATTOS, 1963. 
A presente contribuição consta de uma primbira lista de plantas coletadas na região de São José do Rio Preto e já identificadas até a espécie. Muitas outras plantas, foram, até $o$ presente momento, identificadas até o gênero, e serão, portanto, apresentadas num próximo trabalho.

Qualquer estudo de natureza botânica, na região do Arenito de Bauru, oferece grande interêsse, pois o mesmo abrange cêrca de $25 \%$ da área do Estado de São Paulo, suportando, aproximadamente, $70 \%$ da lavoura cafeeira, $70 \%$ da lavoura algodoeira e boa parte das culturas de arroz e milho (COMISSÃO INTEREST. BACIA PARANA-URUGUAY, 1961).

\section{MATERIAL E MÉTODOS}

Com o auxílio de um jipe doado pela Fundação de Amparo à Pesquisa do Estado de S. Paulo, os, A. A. realizaram excursões semanais de coleta de material, durante $02 .{ }^{\circ}$ semestre de 1962, percorrendo o município de São José do Rio Preto em várias direções. Foram exploradas várias, comunidades de vegetação espontânea, em diversos pontos do município. Também foram coletados materiais ao longo das estradas e nos arredores da cidade, bem como em terrenos cultivados. As espécles coletadas eram levadas ao laboratório, catalogadas e herborizadas, passando a pertencer ao Herbário da Cadeira de Botânica. A determinação das espécies foi feita pelos A. A., na medida das disponibilidades bibliográficas da Cadeira. As que não puderam ser identificadas pelos A. A. foram remetidas para o Instituto de Botânica da Secretaria da Agricultura, onde as determinações foram feitas, pelo Eng. ${ }^{0}$ Agr. ${ }^{\circ}$ João R. de Mattos.

\section{RESULTADOS}

\section{Plantas de São José do Rio Preto}

A presente lista inclui as espécies vasculares (Traqueófitas), por nós coletadas, no $2 .^{\circ}$ semestre de 1962 , na regiāo de São José do Rio Preto, até o momento identificadas.

São, ao todo, 76 espécies, pertencentes a 67 gềneros e 34 famílias. Em se tratando de uma lista parcial, e para facilidade de consulta,todos os nomes (familiais, genéricos e específicos ) estão colocados na ordem alfabética. 
ACANTHACEAE (NEES AB ESENBECK, 1847)

1. Ruellia geminiflora H.B.K.

(= Dipteracanthus geminiflorus H. B. K. = Ruellia hirsuta Vell.).

Planta de lugares muito sêcos; frequentemente pedregosos (NEES AB ESENBECK, 1847)

Venezuela, Bahia, Espírito Santo, Minas Gerais, Rio de Janeiro, Mato Grosso, São. Paulo (NEES AB ESENBECK, 1847)

SJRP-27 (3-10-62)(*)

AMARANTHACEAE (SEUBERT, 1875)

1. Alternanthera ficoidea (L.) R. Br.

( = Gomphrena ficoidea L.)

Colombia, Suriña, Guiana Francesa, Pará (SEUBERT, 1875)

SJRP-112 (13-12-62)

2. Alternanthera brasiliana (L) Kuntnze

( = Gomphrena brasiliana L.)

"Perpétua"

Planta invasora dos cultivos (MONTEIRO, PAIXÃO e MONTEIRO, 1956)

Ceará até Sta. Catarina (SEUBERT, 1875)

SJRP-121 (13-12-62)

Fam. ÀSCLEPIADACEAE (FOURNIER, 1885)

1. Asclepias curassavica L.

"Falsa erva de rato", "Falsa ipecacuanha", "Oficial de sala", "Paina de sapo", "Caga olho".

Planta invasora dos cultivos (CARVALHO, 1959; MONTEIRO, PAIXÃO, MONTEIRO, 1956), venenosa para o Homem e para o gado (KUHLMANN e KUHN, 1947); raíz emética, em pequenas doses (LOFGREN e EVERETT, 1905)

SJRP-69 (12-11-62), SJRP-102 (2-12-62)

(*) número de registro no Herbárlo da Cadeira e data da coleta. 
Fam. BIGNONIACEAE (BUREAU e SCHUMANN, 1896-7)

1. Arrabidaea brachypoda (D.C. ) Bur. et Sch.

Espécie típica do cerrado (RIZZINI; 1963)

Perú, Bolívia, Paraguai, Piaui, Goiás, Bahia, Mato Grosso, Rio de Janeiro, Minas Gerais, São Paulo (BUREAU e SCHUMANN, 1896-7)

SJRP-79 (14-11-62)

2. Bignonia exoleta Vell.

"Batata de caboclo", "Unha de morcego" (KUHLMANN e KUHN, 1947)

Planta comum em terrenos cultivados ( KUHLMANN e KUHN, 1947)

Guianas Britânica, e Francesa, Amazonas, Minas Gerais, Rio de Janeiro, São Paulo. Sta. Catarina. SJRP-44 (17-10-62)

3. Jacaranda caroba P.DC

"Carobinha", "Carobinha do Campo".

Minas Gerais (BUREAU e SCHUMANN, 1896-7), São Paulo

Espécie do cerrado e outras formaçōes (RIZZINI, 1963)

SJRP-17 (25-8-62)

4. Memora axillaris Bur. et $\mathbf{K}$. Sch.

Brasil centro-oeste (BUREAU e SCHUMANN, 1896-7)

SJRP-95 (3-12-62)

5- Tecoma caraiba Mart. ex DC.

( = Tabebuia caraiba (Mart.) Bur.)

"Caraiba", "Pau d'arco do campo" (BUREAU e SCHUMANN, 1896-1897)

Espécie típica do cerrado (RIZZINI, 1963)

Pará, Goiás, Bahia, Minas Gerais, Mato Grosso e São Paulo

SJRP-13 (25-8-62) 
6. Tecoma chrysothrica Mart. ex DC.

"Ipê tabaco", "Pau d'arco amarelo" (BUREAU e SCHUMANN, 1896-7)

Minas Gerais, Espírito Santo; Rio de Janeiro, (BUREAU e SCHUMANN, 1896-7), em São Paulo (LÖFGREN e EVERETT, 1905)

SJRP-23 (12-9162)

CAESALPINIACEAE (BENTHAM, 1859-76)

1. Cassia occidentalis $\mathrm{L}$.

"Fedegoso"

Planta invasora dos cultivos (MONTEIRO, PAIXÃO e MONTEIRO, 1956)

Pará até Paraná (LÖFGREN e EVERETT, 1905)

SJRP-100 (3-12-62), SJRP-110 (13-12-62)

2. Cassia rotundifolia Pers.

Planta invasora dos cultivos (CARVALHO, 1959) México até S. Paulo (LÖFGREN e EVERETT, 1905) SJRP-125 (13-12-62)

3. Cassia rugasa Don.

"Boi gordo"

Espécie típica do cerrado (RIZZINI, 1963)

Piaui até São Paulo (LÖFGREN e EVERETT, 1905)

SJRP-82 (14-11-62)

4. Copaifera Langsdorfii Desf.

"Copaiba", "Óleo de copaiba", "Pau d'óleo"

Espécie do cerrado e de floresta mesófila (RIZZINI, 1963; a óleo contém uma resina medicinal.

Ceará até Paraná (LÖFGREN e EVERETT, 1905) SJRP $\$$ /n (assinalada)

5. Pterogyne nitens Tul.

"Amendoim", "Amendoim bravo", "Amendoim do campo".

Espécie da floresta atlântica e da caatinga 
Ceará, Bahia, Rio de Janeiro (BENTHAM, 1859-76), São Paulo (LÖFGREN a EVERETT, 190.5) Asssinalada

COCHLOSPERMACEAE (EICHLER,1871):

1. Cochlospermum regium (Mart. et Schr.) Pilger

( = Cochlospermum insigne St. Hil.)

"Ruibarbo do campo", "Butuá de corvo"

Espécie típica do cerrado (RIZZINI, 1963)

Pernambuco até São Paulo (LÖFGREN e EVERETT, 1905)

SJRP-16 (25-8-62), SJRP-25 (3-10-62)

COMPOSITAE (BAKER, 1873-84; BARROSO, 1947, 1959 ; LÖFGREN, 1897)

1. Acanthospermum australe $\mathrm{L}$.

( = Acanthospermum xanthioides DC.)

"Carrapicho"

Planta invasora dos cultivos (KUHLMANN e KUHN, 1947; MONTEIRO, PAIXĀO e MONTEIRO, 1956)

Do México e Flórida até a Argentina (BAKER, 1873-84)

SJRP-96 (3-12-62)

2. Bidens pilosa .L

"Picão"

Planta arvense (KUHLMANN e KUHN, 1947)

Regiōes tropicais do mundo (BAKER, 1873-84)

SJRP-113 (13-12-62)

3. Blainvillea rhomboidea Cass.

Regiōes tropicais do mundo (BAKER, 1873-84) SJRP-123 (13-12-62)

4. Eclipta alba (L.) Hassk.

"Lanceta", "Erva de Botão" (BAKER, 1873-84)

Planta invasora dos cultivos (CARVALHO, 1959); já tida como remédio contra mordeduras (BAKER, 1873-84) 
Regiōes tropicais do mundo (BAKER, 1873-84)

SJRP-103 (3-12-62), SJRP-107 (13-12-62)

5. Gochnatia paniculata (DC.) Cabr.

Planta campestre (BAKER, 1873-84)

Brasil oriental (BAKER, 1873-84)

SJRP-7 (19-8-62)

6. Parthenium hysterophorus L.

Planta ruderal

México e Ilhas do Caribe (naturalizada em quase tôda América e partes do Velho Mundo)

SJRP-101 (3-12-62)

7. Porophyllum ruderale Baker

"Couve-cravinho", "Erva freșca" (LÖFGREN e EVERETT, 1905)

América tropical

Planta ruderal (BAKER, 1873-84), invasora dos cultivos (CARVALHO, 1959; MONTEIRO, PAIXÃO e MONTEIRO, 1956).

SJRP-106 (13-12-62)

8. Tagetes minuta $\mathrm{L}$.

"Cabo de rojão", "Coarí bravo"

Espécie arvense (KUHLMANN e KUHN, 1947), tida como remédio anti-reumático

Minas Gerais, Rio de Janeiro, São Paulo, Sta. Catarina, Paraguai, Argentina, Uruguai (BAKER, 1873-84)

SJRP-s/n (Assinalada)

CONVOLVULACEAE (MEISSNER, 1869-71)

1. Jaquemontia hirsuta Chois.

México, Perú, Guiana Britânica, Amazonas, Bahia, Goiás, Minas Gerais, São Paulo (MEISSNER, 1869, 1871)

SJRP-85 (19-11-62)

2. Merremia glabra (Chois.) Hall

Planta arvense (KUHLMANN e KUHN, 1947) 
Venezula, Guianas, Pará, Minas Gerais, Espírito Santo, Rio de Janeiro, São Paulo, Perú

SJRP-97 (3-12-62)

Fam. EBENACEAE (MIQUEL, 1856)

1. Diospyros camporum Warm.

( = Diospyros hispida DC., var. camporum Warm ( RIZZINI, 1963)

Espécie típica do cerrado (RIZZINI, 1963)

Minas Gerais (MIQUEL, 1856), São Paulo

SJRP-36 (14-10-62), SRJP-63 (1-11-62)

Fam. ERYTHROXYLACEAE (PEYRITSCH, 1878)

1. Erythroxylon cuneifolium (Mart.) O. E. SCHULZ "Mercúrio do campo", "Galinha choca" (KUHLMANN e KUHN, 1947)

SJRP-62 (1-11-62)

Fam. GRAMINEAE (DOELL, 1871-83)

1. Brachiaria plantaginea (Link.) Hitch

"Capim marmelada"

Planta invasora dos cultivos (CARVALHO, 1959)

Goiás, Bahia, Minas Gerais, Rio de Janeiro, S. Paulo (DOELL, 1871-83)

SJRP-119 (13-12-62)

2. Cynodon dactylon (L.) Pens.

"Capim Bermuda", "Capim de burro", "Capim pé de galinha"

Planta arvense e ruderal.

Orig. Europa e Ásia; no Brasil: Amazonas, Pará, Piaui, Bahia, Minas Gerais, Rio de Janeiro, Sta. Catarina (DOELL, 1871-83), S. Paulo

SJRP-120 (13-12-62)

3. Setaria geniculata Beauv.

SJRP-111 (13-12-62) 
4. Trichachne insularis (L.) Nees

( = Andropogon insularis L.)

Pará, Ceará, Piauí, Goiás, Bahia, Minas Gerais, Espírito Santo, Rio de Janeiro, Sta. Catarina (DOELL, 1871-83), S. Paulo.

SJRP-116 (13-12-62)

5. Tricholaena teneriffae (L. f.) Pavl.

"Capim favorito"

Planta invasora dos cultivos (CARVALHO, 1959)

Orig.: África, naturalizada no Brasil

SJRP-118 (13-12-62)

Fam. LABIATE (SCHMIDT, 1858)

1. Hyptis suaveolens Poir.

"Mentrasto grande" (LÓFGREN e EVERETT, 1905) Planta invasora dos cultivos (CARVALHO, 1959; MONTEIRO, PAIXÃO e MONTEIRO, 1956)

Pará, Bahia, Minas Gerais, Rio de Janeiro (SCHMIDT, 1858), Goiás, São Paulo (LÖFGREN e EVERETT, 1905).

SJRP-124 (13-12-62)

Fam. LYCOPODIACEAE (SPRING, 1840)

1. Lycopodium cernuum L.

"Licopódio"

Planta das matas sêcas (SPRING, 1840)

Regiōes tropicais (SPRING, 1840)

SJRP-22 (12-9-62)

Fam. MALVACEAE (GÜRKE, 1892; SCHUMANN, 1891-2)

1. Abutilon indicum Sweet

América central, Antilhas, Amazonas, Guiana Holandesa, Perú, naturalizado na Ásia e África, tropicais (SCHUMANN, 1891-2)

SJRP-130 (13-12-62) 
2. Urena lobata L., var. americana Gurke "Guaxuma”, “Carrapicho", "Malva rosa" Planta invasora dos cultivos (CARVALHO, 1959) Antilhas, Guianas, Pernambuco, Bahia, Minas Gerais, Rio de Janeiro, Sta. Catarina (GÜRKE, 1892), São Paulo, Nat. na África Tropical. SJRP-21 (9-9-62)

Fam. MELIACEAE (DE CANDOLLE, 1878)

1. Guarea pohlli C.DC.

"Marinheiro"

Minas Gerais (DE CANDOLLE, 1878)

SJRP-67 (12-11-62)

Fam. MENISPERMACEAE (EICHLER, 1864)

1. Cissampelos glaberrima St. Hil

"Erva de N. Senhora", (LÖFGREN e EVERETT, 1905), "Butinha", Cipó de Cobra", "Parreira caa-peba" (KUHLMANN e KUHN, 1947)

Minas Gerais, Mato Grosso, Rio de Janeiro (EICHLER, 1864), São Paulo e Paraná (LÓFGREN e EVERETT, 1905)

SJRP-43 (21-10-62), SJRP-64 (1-11-62)

Fam. MIMOSACEAE (BENTHAM, 1859-76)

1. Mimosa subserica Benth.

Minas Gerais, Rio de Janeiro, São Paulo, Brasil meridional (BENTHAM, 1859-76)

SJRP-26 (3-10-62)

2. Piptadenia peregrina Benth.

"Paricá" (BENTHAM, 1859-76), "Angico do Campo" Colombia, Guiana, Rio Branco, Amazonas, Goiás, Rio de Janeiro, São Paulo (BENTHAM, 1859-76). SJRP-20 (9-9-62). 
3. Plathymenia reticulata Benth.

( = Chrysoxylon vinatico Casar.)

"Vinhático do campo", "Candeia", "Amarelo"

Espécie típica do cerrado (RIZZINI,1963)

Bahia, Goiás, Minas Gerais, São Paulo (BENTHAM, 1859-76)

Assinalada.

Fam. MYRTACEAE (BERG, 1857-8)

1. Campomanesia obversa Berg.

( = Psidium obversum Miq.)

"Guabiroba" (BERG, 1857-8), "Gabiroba" (loc.)

Espécie típica do cerrado (RIZZINI, 1963); frutos comestiveis

Minas Gerais (BERG, 1857-8), São Paulo

SJRP-28 (3-10-62), SJRP-34 (14-10-62)

2. Hexachlamys minarum Legr.

SJRP-6 (15-8-62)

OCHNACEAE (ENGLER, 1876)

1. Ouratea spectabilis (Mart.) Engl.

"Farinha sêca"

Espécie típica do cerrado (RIZZINI, 1963)

Minas Gerais, Mato Grosso, São Paulo (ENGLER, 1876)

SJRP-38 (14-10-62)

ONAGRACEAE (MICHELI, 1875)

1. Jussiaea caparosa Camb.

"Cruz de Malta"

Brasil Central e Meridional (MICHELI, 1875)

SJRP-70 (12-11-62) 
OXALIDACEAE (PROGEL, 1877)

1. Oxalis physocalyx Zucc.

"Azedinha"

Goiás, São Paulo (LÖFGREN e EVERETT, 1905)

SJRP-58 (1-11-62), SJRP-99 (3-12-62)

PAPILIONACEAE (BENTHAM, 1859-76)

1. Andira humilis Mart.

Espécie típica do cerrado (RIZZINI, 1963)

Brasil meridional (BENTHAM, 1859-76), Minas Gerais e São Paulo (LÖFGREN e EVERETT, 1905)

SJRP-24 (3-10-62)

2. Bowdichia virgilioides Benth.

"Sebipira" (BENTHAM, 1859-76), "Sucupira parda" Espécie do cerrado e outras formaçōes (RIZZINI, 1963)

Venezuela, Guianas, Pará, Goiás, Bahia, Minas Gerais, Brasil meridional (BENTHAM, 1859-76)

SJRP-19 (9-9-62)

3. Platypodium elegans Vog.

"Amendoim do cerrado", "Jacarandá bravo"

Espécie do cerrado e outras formações (RIZZINI, 1963)

Bahia, Goiás, Mato Grosso, Minas Gerais (BENTHAM, 1859-76), S. Paulo

SJRP-42 (14-10-62)

4. Pterodon pubescens Benth

"Faveiro", "Sucupira lisa"

Espécie do cerradó e outras formações (RIZZINI, 1963)

Bahia, Goiás, Mato Grosso, Minas Gerais e Brasil meridional (BENTHAM, 1859-76)

Assinalada. 
5. Stylosanthes viscosa Suv.

"Meladinha", "Alfafa viscosa" (KUHLMANN e KUHN, 1947)

Planta dos campos arenosos e sêcos (BENTHAM, 1859-76)

América Central, Antilhas, Guianas, Brasil, Uruguai (BENTHAM, 1859-76)

SJRP-108 (13-11-62)

6. Sweetia elegans (Vog.) Bentham

"Perobinha", "Perobinha do campo"

Espécie típica do cerrado (RIZINI, 1963) ; tida como remédio antipirético e antissifilítíco (LÖFGREN e EVERETT, 1905)

Mato Grosso, Minas Gerais, São Paulo (BENTHAM, 1859-76)

SJRP-46 (25-10-62), SJRP-51 (1-11-62)

Fam. POLYGONACEAE (MEISSNER, 1855)

1. Coccoloba paniculata Meissn

"Cabuçú" (LÖFGREN e EVERETT, 1905), "Canela de indio" (loc.)

Amazonas, Goiás, Mato Grosso (MEISSNER, 1855), São Paulo (LÖFGREN e EVERETT, 1905)

SJRP-50 (25-1-62)

Fam. PONTEDERIACEAE (SEUBERT, 1847)

1. Eichornia crassipes (Mart.) Solms

"Aguapé"

Planta aquática

Guianas, Minas Gerais, Brasil meridional (SEUBERT, 1847)

SJRP-83 (14-11-62)

Fam. RHAMNACEAE (REISSEK, 1861)

1. Gouania mollis Reiss

Brasil meridional (REISSEK, 1861)

SJRP-80 (14-11-62) 
Fam. RUBIACEAE (MÚLLER, 1881; SCHUMANN, 1888-9)

1. Diodia setigera DC

"Poaia"

Bahia, Espírito Santo, Minas Gerais, São Paulo (SCHUMANN, 1888-9)

SJRP-122 (13-12-62)

2. Palicourea rigida H.B.K.

( = Psycotria rigida Willd.)

"Douradinha", "Douradão", "Gritadeira".

Espécie típica do cerrado (RIZZINI, 1963)

Venezuela, Guianas, Brasil tropical, até São Paulo (MÚLLER, 1881)

SJRP-83 (14-11-62)

3. Tocoyena formosa $\mathrm{K}$. Sch.

"Genipapo bravo", "Genipapo do campo" (SCHUMANN, 1888-9)

Espécie do cerrado e outras formaçōes (RIZZINI, 1963)

Guiana Britânica, Maranhão, Bahia, Alagôas, Pernambuco, Minas Gerais, São Paulo, Mato Grosso ( SCHUMANN, 1888-9)

SJRP-59 (1-11-62)

Fam. SAPINDACEAE (RADLKOFFR, 1892-1900)

1. Matayba arborescens Radlk.

"Maca-apa-ipu" (RADLKÓFER, 1892-1900)

Guiana, Amazonas, Pará, (RADLKÓFER, 18921900), São Paulo

SJRP-53 (1--1-62)

2. Matayba guianensis Aubl.

"Cambotá" (LÖFGREN e EVERETT, 1905), "Cambotá branco" (RADLKÖFER, 1892-1900), "Cambotá bravo" (RADLKÖFER, 1892-1900)

Guianas, Perú, Amazonas até Minas Gerais (RADLKÓFER, 1892-1900) e São Paulo

SJRP-31 (14-10-62) 
3. Matayba guianensis - forma micrantha

Perú, Mato Grosso, Goiás, Minas Gerais, Rio de Janeiro, São Paulo

SJRP-18 (25-8-62)

Fam. SAPOTACEAE (MIQUEL, 1863)

1. Chrysophyllum gonocarpum (Mart. et Eichl.) Engl. ( = Sapota gonocarpa Mart. et Eichl.)

"Guatambu", "Guatambu de leite" (KUHLMANN e KUHN, 1947), "Peroba branca" (KUHLMANN e KUHN, 1947; LÖFGREN e EVERETT, 1905)

Planta da floresta atlântica (MIQUEL, 1863)

Rio de Janeiro, São Paulo (LOFGREN e EVERETT, 1905)

SJRP-45 (17-10-62)

Fam. STERCULIACEAE (SCHUMANN, 1886)

1. Helicteres ovata Lam.

"Sacarrolha", "Sete voltas", "Rosca de mula"

"Espécie típica do cerrado (RIZZINI, 1963)

Goiás, Minas Gerais, Rio de Janeiro, São Paulo (SCHUMANN, 1886)

SJRP-41 (14-10-62)

2. Waltheria americana $\mathrm{L}$.

"Malva branca" (KUHLMANN e KUHN, 1947), "Malva veludo" (KUHLMANN e KUHN, 1947)

Planta invasora dos cultivos (CARVALHO, 1959; MONTEIRO, PAIXÃO e MONTEIRO, 1956)

Regióes tropicais, Brasil todo (SCHUMANN, 1886) SJRP-77 (14-11-62)

Fam. STYRACACEAE (PROGEL, 1868)

1. Styrax camporum Pohl.

"Estoraque do campo" (PROGEL, 1868), "Estoraqueiro", Pau de remo" (KUHLMANN e KUHN, 1947) 
Espécie do cerrado e outras formações (RIZZINI, 1963)

Minas Gerais, S. Paulo, Paraná (LÖFGREN e EVERETT, 1905)

SJRP-57 (1-11-62)

Fam. TILIACEAE (SCHUMANN, 1886)

1. Luhea divaricata Mart.

"Açoita-cavalos", "Açoita-cavalos branco" (SCHUMANN, 1886)

Espécie do cerrado e outras formaçōes (RIZZINI, 1963)

Goiás, Minas Gerais, Rio de Janeiro, São Paulo, Paraguai, Uruguai, Argentina (SCHUMANN, 1886) SJRP-98 (3-12-62)

2. Luhea uniflora St. Hil

"Açoita-cavalos"

Bolivia, Bahia, Minas Gerais, Rio de Janeiro, São Paulo (SCHUMANN, 1886)

SJRP-65 (1-11-62)

VERBENACEAE ('SCHUMANN, 1851)

1. Aegiphila Lhotzkiana Cham.

"Tamanqueiro"

Espécie típica do cerrado (RIZZINI, 1963)

Bahia, M. Gerais, (SCHAUER, 1851), São Paulo (LOFGREN e EVERETT, 1905)

SJRP-81 (14-11-62)

2. Aegiphila verticillata Vell.

( = Aegiphila tomentosa Cham.

Minas Gerais, São Paulo (SCHAUER, 1851)

SJRP-32 (14-10-62)

3. Lantana camara L.

"Camará verdadeira" (KUHLMANN e KUHN, 1947), "Camará" (LÖFGREN e EVERETT, 1905), "Cambará"

Antilhas, Venezuela, Guianas, Minas Gerais, Rio de 
Janeiro (SCHAUER, 1851), São Paulo e Paraná (LOFGREN E EVERETT, 1905)

SJRP-5 (15-8-62), SJRP-54 (1-11-62)

4. Lantana camara L., var. aculeata (L.) Moldenke

"Cambará de espinho"

Planta invasora dos cultivos (CARVALHO, 1959;

MONTEIRO, PAIXÃO e MONTEIRO, 1956)

SJRP-75 (14-11-62)

5. Lantana trifolia $\mathrm{L}$.

"Camará", " Milho de grilo", "Cangica" (KUHLMANN e KUHN, 1947)

Planta invasora dos cultivos, (CARVALHO, 1959; MONTEIRO, PAIXÃO e MONTEIRO, 1956) seg. formações (RIZZINI, 1963), dos monturos (KUHLMANN e KUHN, 1947)

Antilhas, Guianas, Venezuela, Perú, M. Gerais e S. Paulo (SCHAUER, 1851)

SJRP-104 (3-12-62)

6. Stachytarpheta australis Moldenke

"Gervão"

Planta arvense (KUHLMANN e KUHN, 1947)

SJRP-109 (13-12-62)

VIOLACEAE (EICHLER, 1871-6)

1. Hybanthus communis Taub.

Planta do subosque das capoeiras úmidas (KUHLMANN e KUHN, 1947)

Bahia até S. Paulo (LÖFGREN e EVERETT, 1905) SJRP-71 (12-11-62)

Fam. VOCHYSIACEAE (WARMING, 1875)

1. Qualea grandiflora Mart.

"Pau-terra"

Espécie típica do cerrado (RIZZINI, 1963)

Brasil Central (WARMING, 1875) até São Paulo (LÖFGREN E EVERETT, 1905)

SJRP-86 


\section{CONCLUSÃO}

Das 76 espécies apresentadas, nesta lista, 14 são exclusivas do cerrado, 9 ocorrem nêle e em outras formações, 20 são plantas invasoras (arvenses ou ruderais), e as restantes não foram ainda classificadas, em relção à sua distribuição ecológica.

Embora esta primeira lista não seja bastante ampla para poder ser considerada como representativa da flora da região de São José do Rio Preto, revela desde já, o caráter heterogêneo da mesma, tanto no que se refere às formações vegetais, como em relação à origem geográfica de seus componentes.

\section{AGRADECIMENTOS}

Deixamos aqui consignados os nossos agradecimentos à Fundação de Amparo à Pesquisa do Est. de São Paulo, pelo auxílio que nos concedeu, e ao Eng. Agr. João Rodrigues, de Mattos, da Seção de Fanerógamos do Instituto de Botânica de São Paulo, pela colaboração que nos prestou, na identificação taxonômica das espécies para lá remetidas.

\section{SUMMARY}

The authors present the first list of vascular plants collected by them in the region of São José do Rio Preto county, State of São Paulo, Brazil. This list of 76 species includes 23 ones of cerrado (14 of these exclusively of cerrado), and 20 species of adventitious plants (arvenses or ruderales). The 76 species are distributed in 67 genera and 34 families.

The climate of the county is mesothermic, tending to megathermic, corresponding to the Koppen's Cwa type. The predominant soil is a dark-red latosol, originated from decomposition of Arenito de Bauru (Bauru sandy soil of upper Cretaceous).

\section{BIBLIOGRAFIA CITADA}

BAKER, J. G. 1873-76-82-84 - Compositae, in C. F. P. Von Martius et A. G. Von Martius et A. G. Eichler Flora Brasillensis Vol. VI, II-III, Monachil. 
BARRoso, G. M., 1947 - Chave para a Determinação de Gêneros İndigenas e Exóticos das Compositae no Brastl. Rodriguésia, Ano $\mathrm{X}, \mathrm{n} .^{\circ}$ 21, Rio de Janeiro, Brasil.

_. 1959 - Flora da Cidade do Rio de Janeiro. Compositae. Rodriguésia, Anos XXI e XXII, n.. 33-34. Rio de Janeiro, Brasil.

BENTHAM, G., 1859-62-70-76 - Leguminosae, in C.F.P. Von Martius et A. G. Eichler Flora Brasiliensis Vol. XV, I-II-III, Monachii.

BERG, O., 1857-58 - Myrtaceae, in C.F.P. Von Martius et A. E. Eichler Flora Brasiliensis Vol. XIV, I. Monachii.

BUREAU, E. \& C. SCHUMANN, 1896-97 - Bignoniaceae, in C.F.P. Von Martius et A. G. Eichler Flora Brasiliensis Vol. VIII, II. Monachii.

CARVALHO, LUIZ F. de 1959 - Plantas Invasoras de Culturas no Estado do Rio de Janeiro, 2. ${ }^{\circ}$ Semin. Bras. Herb. e Ervas daninhas, Belo Horizonte, 1958. Centro Nac. Ens. Pesq. Agronômicas Rio de Janeiro, Brasil.

COMISSÃO INTERESTADUAL DA BACIA PARANA-URUGUAI, 1961 - Solos da Bacia Paraná-Urruguai. São Paulo, Brasil.

DE CANDOLLE, G., 1878 - Meliaceae, in C.F.P. Von Martius et A. G. Eichler Flora Brasiliensis Vol. XI, I. Monachii .

DOELL, J. C, 1871-77-78-80-83 - Gramineae, in C.F.P. Von Martius et A. G. Eichler. Flora Brasiliensis, Vol. II, III. Monachii.

EICHLER, A. G., 1864 - Menispermaceae, in C.F.P. Von Martius et A. G. Eichler. Flora Brasiliensis, Vol. XIII, I. Monachii.

$$
\text { 1871-a. - Bixaceae, in C.F.P. Von Martius et }
$$

A. G. Eichler. Flora Brasiliensis, Vol. XIII, I. Monachii.

A. G. Eichler, Flora Brasiliensis, Vol. XIII, I. Monachil.

ENGLER, A., 1876 - Ochnaceae, in C.F.P. Von Martius et A. G. Eichler. Flora Brasiliensis, Vol. XII, II. Monachii.

FOURNIER, E., 1885 - Asclepiadaceae, in C.F.P. Von Martius et A. G. Eichler. Flora Brasiliensis, Vol. VI, IV. Monachil.

GÜRKE, M., 1892 - Malvaceae, in C. F. P. Von Martius et A. G. Eichler. Flora Brasiliensis, Vol. II, III. Monachili.

KUHLMANN, M. \& E. KUHN, 1947 - A Flora do Distrito de Ibiti. Inst. Bot. São Paulo, Brasil.

LöFGREN, A., 1897 - Flora Paulista, I. Familia Compositae. Comm. Geogr. Geol. de São Paulo, Bol. n. ${ }^{\circ}$ 12, 1.496. São Paulo, Brasil. São Paulo, Brasil. \& C. H. L. EVERETT, 1905 - Analysis de Plantas,

MEISSNER, C. F., 1855 - Polygonaceac, in C.F.P. Von Martius et A. G. Eichler. Flora Brasiliensis, Vol. V, I. Monachil.

et A. G. Eichler. Flora Brasiliensis, Vol. VII. Monachii. 
MICHELI, M., 1875 - Onagraceae, in C.F.P. Von Martius et A. G. Eichler. Flora Brasiliensis, Vol. XIII, II. Monachii.

MIQUEL, F. A. G., 1856 - Ebenaceae in C.F.P. Von Martius et A. G. Eichler. Flora Brasiliensis, Vol. VII. Monachii.

MONTEIRO, Fo, H., J. C. PAIXÃO \& J. M. MONTEIRO, $1956-$ Plantas Herbáceas Invasoras de Cultivos 1. Semin. Bras. Herb. e Hervas Daninhas, Rio de Janeiro, 1956. Centro Nac. Ens. Pesq. Agronômicas. Rio de Janeiro, Brasil.

MttLLER, ARG. J., 1881 - Rubiaceae, in C. F. P. Von Martius et A. G. Eichler. Flora Brasiliensis, Vol. VI, V. Monachii.

NEES AB ESENBECK, C. G., 1847 - Acanthaceae, in C.F.P. Von Martius et A. G. Eichler. Flora Brasiliensis, Vol. IX. Monachii.

PEYRITSCH, J., 1878 - Erythroxylaceae, in C.F.P. Von Martius et A. G. Eichler. Flora Brasiliensis, Vol. XII, I. Monachii.

PROBEL, A., 1877 - Oxaltdaceae, in C.F.P. Von Martius et A. G. Eichler. Flora Brasiliensis, Vol. XII, II. Monachii.

1868 - Loganiaceae, in C.F.P. Von Martius et A. G. Eichler. Flora Brasiliensis, Vol. VI, I. Monachii.

RADLKÖFER, L. 1892-97-1900 - Sapindaceae ,in C. F.P.Von Martius et A. G. Eichler. Flora Brasiliensis, Vol. XIII, III. Monachii.

REISSEK, S. 1861 - Thamneae, in C.F.P. Von Martius et A. G.

Eichler. Flora Brasiliensis, Vol. XI, I. Monachii.

RIZZINNI, C. T., 1963 - A Flora do Cerrado. Análise florística das savanas centrais. Simpósio sôbre o Cerrado, São Paulo 1962. São Paulo, Brasil.

SCHAUER, J. C. 1851 - Verbenaceae, in C.F.P. Von Martius et A. G. Eichler. Flora Brasiliensis, Vol. IX. Monachii.

SCHMIDT, J. A, 1858 - Labiatae, in C.F.P. Von Martius et A. G. Eichler. Flora Brasiliensis, Vol. VIII, I. Monachii.

SCHUMANN, C., 1886-a. - Sterculiaceae in C. F. P. Von Martius et A. G. Eicheler. Flora Brasiliensis, Vol. XII, III. Monachii. 1886-b. - Tiliaceae, in C. F. P. Von Martius et A. G. Eichler. Flora Brasiliensis, Vol. XII, III. Monachii. 1888-89 - Rubiaceae, in C.F.P. Von Martius et A. G. Eichler. Flora Brasiliensis, Vol. VI, VI. Monachii. 1891-92 - Malvaceae, in C.F.P. Von Martius et A. G. Eichler. Flora Brasiliensis, Vol. XII, III. Monachii.

SEUBERT, M., 1847 - Pontederiaceae, in C. F. P. Von Martius et A. G. Eichler. Flora Brasiliensis, Vol. III, I. Monachii.

1875 - Amarantaceae, in C.F.P. Von Martius et A. G. Eichler. Flora Brasiliensis, Vol. V, I. Monachii.

SPRING, A. F. 1840 - Lycopodineae, in C.F.P. Von Martius et A. G. Eichler. Flora Brasiliensis, Vol. I, II. Monachil.

WARMING, E. 1875 - Vochysiaceae, in C.F.P. Von Martius et A. G. Eichler. Flora Brasiliensis, Vol. XIII, II. Monachii. 
